\title{
Argumentation during active learning strategies in a SCALE-UP environment
}

\author{
Esmeralda Campos ${ }^{1}$, Leonor Silva ${ }^{2}$, Silvia Tecpan ${ }^{3}$, and Genaro Zavala ${ }^{1}$ \\ ${ }^{I}$ Department of Physics, Tecnologico de Monterrey, Av. Eugenio Garza Sada 2501, Monterrey, Mexico, 64849 \\ ${ }^{2}$ Online Programs, Tecnologico de Monterrey, Av. Eugenio Garza Sada 2501, Monterrey, Mexico, 64849 \\ ${ }^{3}$ Physics Department, Universidad de Santiago de Chile, Av. Ecuador 3493, Santiago, Chile, 9170124
}

\begin{abstract}
In this study, we explore students' discussions during active learning strategies of Peer Instruction and Tutorial-like activities in a SCALE-UP environment. Discussions were recorded in one of the tables of a student-centered learning room in Mexico, and analyzed through a qualitative approach using Toulmin's argumentation pattern as a framework of reference. We found that the circular table functions as a learning community in the SCALE-UP environment, however, the dynamics of argumentation of the table are different while implementing each learning strategy. During Tutorial-like activities, students center their discussions on each small team, while during peer instruction students center their discussion around the whole table. These findings provide an insight into the kind of competencies that we mean to develop in our students by engaging them in active learning strategies in student centered learning environments.
\end{abstract}

\section{INTRODUCTION}

There is an ongoing discussion in Physics Education Research about the role that argumentation plays in the development of scientific reasoning and the learning of abstract concepts. Research done in this context suggests that through argumentation students have the opportunity to confront new physics concepts to their own alternative conceptions and correct them $[1,2]$. Active learning strategies such as Peer Instruction (PI) and Tutorials, which are strongly associated to argumentation, are increasingly being promoted and implemented in physics education [3].

The learning environment also plays a role in the dynamics of argumentation that take place during active learning strategies. For example, when creating a discussion in a traditional classroom, students may feel limited to interact only with the people sitting to either side of their seat, while in a SCALE-UP environment students can form a learning community at the table. A learning community consists of a group of people with different levels of expertise and a common goal of expanding knowledge, and is desirable for promoting retention and persistence in students [4].

Argumentation happening during active learning strategies such as PI has been studied in the context of Mechanics in traditional environments [5, 6]. Research on discussions during PI has been conducted mainly in massive auditorium-like classrooms where students often interact with the people sitting next to them. Even though these studies give a broad insight into the effectiveness of such strategy, they fail to study the effect of the learning environment on the students' discussions. While PI is broadly used in traditional environments such as auditorium-like classrooms, it is also useful in active learning environments such as SCALE-UP.

Tutorials for Introductory Physics are widely used and proven to be effective in tutorial workshops [3, 7]. In contrast, Tutorials have proven to sacrifice their effectiveness in SCALE-UP environments possibly due to the ratio of students/instructors [8]. As a result, Beichner and Saul suggest implementing Tutorial-like activities by breaking down tutorials into smaller tasks and modifying them as needed; students in SCALE-UP environments interact with each other and with the instructors while working on Tutorial-like activities [8]. This study seeks to contribute by exploring the dynamics of argumentation that take place during PI and Tutorial-like activities in a SCALE-UP environment.

\section{BACKGROUND}

Argumentation plays a key role in active learning strategies, such as PI and Tutorial-like activities. These strategies allow students to evaluate previous alternative conceptions and, through discussions with their peers, create new concepts. According to Mazur, discussion with peers helps students to understand conflictive concepts better because some of their peers have already overcome the same conflict and are ready to show the reasoning behind it, and also because the language that students use to communicate with each other is the same, but different from the language that the professor uses with students [9]. 


\section{A.Toulmin's argumentation pattern}

A model of argumentation that has been increasingly used in Science Education is Toulmin's argumentation pattern [2]. This model identifies the basic components of an argument as claim, grounds, warrants and backing; qualifiers and rebuttals are identified as additional elements in complex arguments. For further detail in these elements refer to Driver et al. [2]. Although these elements serve the purpose of identifying an argument, they fail to assess the veracity of the conclusions [2]. Therefore, learning through argumentation also needs to take into account social factors, such as context and interactions, and to qualitatively analyze the discussions performed by a group of people.

\section{B.Peer instruction}

PI refers to the education technique introduced by Mazur in which students interact to solve a conceptual question [9]. The instructor presents a multiple choice question to the students, who choose their answer individually and vote anonymously. The instructor then asks the students to find someone whose answer was different from theirs and to convince that person of their answer. After peer discussion, the poll is reopened and students vote for a second time; the instructor then gives a conclusion reassuring their knowledge. For further detail about this learning strategy refer to Mazur [9].

Discussions during PI have been largely studied in traditional environments, mainly in the context of Mechanics. Crouch and Mazur proved that PI promotes conceptual reasoning and problem solving abilities in introductory physics courses [5]. Wood, Galloway, Hardy and Sinclair studied students' discussions, which revealed that students use three types of resources linked to reasoning within their dialogues: previous knowledge, relations between knowledge elements, and control structure [6].

\section{C.Tutorial-like activities}

Tutorial-like activities are worksheets created by the instructor with the objective of guiding the student step by step on the construction of new concepts, they are strongly based on model problem solving, and sometimes resolve a cognitive conflict; these worksheets are inspired on Tutorials for Introductory Physics [7]. Each activity has a specific objective for which questions are made at the beginning with an introductory approach, and new concepts are incorporated at an adequate pace.

Even though these activities are answered in writing, they require an oral discussion for students to reach a consensus. Students work collaboratively, exchanging ideas and reasoning that lead to the answer of the question in turn. The instructor supervises students' performance by asking them questions to make sure they are working on the right direction; the instructor also asks key questions to lead them into the objective of the activity. Once most teams have completed the activity, the instructor gives closure by reviewing the concepts, reassuring learning.

\section{D.Student centered learning environment}

SCALE-UP environments are designed to naturally promote discussion, making it adequate for active learning strategies when argumentation is essential. The SCALE-UP environment has been set up in Mexico in a private university in the northern part of the country, and is known as Student Centered Learning room (ACE room for its acronym in Spanish). Besides being a SCALE-UP environment, the ACE room has a research table that is equipped with three video cameras and three microphones that allow the researcher to record the interactions that happen on each team of that table [10].

Considering the reviewed literature, when using these two learning strategies in a SCALE-UP environment, the hypothesis is that either of the following behaviors is expected: (1) students' discussions have a similar dynamic around the learning community on both strategies, or (2) students create a different discussion dynamic during PI than that during Tutorial-like activities. Based on this hypothesis, the following research question is formulated: How are the interactions that take place during active learning activities, such as PI and Tutorial-like activities, in a SCALE-UP environment?

\section{METHODOLOGY}

Participants. The participants were nine undergraduate students enrolled in an Electricity and Magnetism (EM) course taking place in the ACE room of the university. The setting was three teams of three students each around one table of the room. The teams were formed based on their performance on the first midterm evaluation of the course by combining one average student, one above-average student and one below-average student, without considering the students located on the extremes, who performed best and worst on the evaluation. The students located on the extremes were seated at other tables in the classroom and were not considered in this study.

Figure 1 represents the distribution of participants sitting around the table, which was consistent throughout the duration of the study. To respect the confidentiality of the participants, we used labels to identify them: capital letters correspond to the team and small caps represent their physical place around the table; thus the participant from team $A$ sitting on chair $a$ is labeled as $A a$. These labels are used throughout the text and are independent of students' performance. 


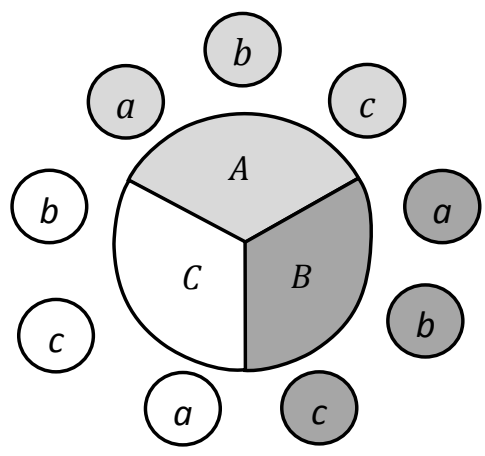

FIG. 1. Distribution of participants in the research table of the ACE room.

Data collection. The environment described is part of the equipment used for observation; the students sat at the research-technology enabled table of the room, and their conversations and interactions were recorded using three video cameras and three microphones, one for each small team seated around the table; a computer was used to keep the recordings. The main instrument of this study is observation of the discussions, which were analyzed through a qualitative and phenomenological approach, using Toulmin's argumentation pattern as reference. Not all discussions that took place during observation followed Toulmin's argumentation pattern and thus could not be considered arguments under this framework. Only discussions that followed Toulmin's model of argumentation were transcribed and analyzed.

Table I provides a description of the role that each student took on discussions. Their participation behavior was repeatedly observed during arguments. The labels used to identify students on Table I correspond to their sitting arrangement as represented on Fig. 1. As it can be observed from Table I, some students took a Leader (LD) role, other students an Active Participant (AP) role, while others a Passive Participant (PP) role. The description for each of these roles is based on the elements of Toulmin's model of argumentation.

\section{RESULTS}

Active learning strategies are greatly based on student involvement and interactions, while keeping the student active. As a result, different types of interactions are observed for different learning strategies. Tutorial-like activities allow the students to interact within their small teams, centering the discussion upon the leader of each team. There are three ongoing discussions at the table and occasional interactions with other teams or the instructor, as to reassure a specific question or topic. This dynamic is represented in Fig. 2, where the interactions of the leaders of each small team are highlighted with bold arrows. Thus, for Tutorial-like activities, a learning community of nine
TABLE I. Description of the roles that each student took on discussions; student labels are assigned according to the distribution shown in Fig. 1, and roles are described using Toulmin's argumentation pattern as reference framework.

\begin{tabular}{|c|c|c|}
\hline Student & Role & Description \\
\hline$A a$ & AP & $\begin{array}{l}\text { Backed claims with data, rebutted } \\
\text { previous claims. }\end{array}$ \\
\hline$A b$ & LD & $\begin{array}{l}\text { Made initial claims, set grounds for } \\
\text { discussion. }\end{array}$ \\
\hline$A c$ & PP & Contributed personal opinions. \\
\hline$B a$ & LD & $\begin{array}{l}\text { Made initial claims, set grounds, made } \\
\text { extensive use of qualifiers. }\end{array}$ \\
\hline$B b$ & AP & Rebutted previous claims. \\
\hline$B c$ & AP & $\begin{array}{l}\text { Backed claims with grounds and } \\
\text { warrants; data and } \\
\text { formulas/procedures. }\end{array}$ \\
\hline $\mathrm{Ca}$ & PP & Did not contribute to the discussions. \\
\hline $\mathrm{Cb}$ & PP & Asked questions. \\
\hline$C c$ & LD & $\begin{array}{l}\text { Made initial claims, set grounds for } \\
\text { arguments and backed claims with } \\
\text { data and warrants. }\end{array}$ \\
\hline
\end{tabular}

people is given by the table with three ongoing discussions centered on each small team $[4,10]$.

During PI the discussion is found to be centered on the table, instead of on small teams; the leaders of each small team and the active students are constantly involved in the discussion, which yields to a whole consensus about the second answer. This dynamic is represented in Fig. 3. The table again acts as a learning community of nine people, but with a different dynamic compared with what is seen during Tutorial-like activities. It is worth mentioning that the arrows in Fig. 3 represent one central discussion happening at the table, involving all or some of the above mentioned leaders and active participants, with no explicit distinction in their behavior. This means that leaders and active students participated in the discussion, and it was not necessarily centered on the leaders.

\section{DISCUSSION}

To answer the main question posed in this study, it was found that students create different argumentation dynamics during PI compared with Tutorial-like activities. These dynamics are established within the learning community given by the table in both cases, but the interactions are different for each learning strategy. The 7-foot diameter tables in a SCALE-UP environment are specifically designed to allow students to comfortably interact with all their peers sitting around it; therefore, both types of interactions are directly influenced by the environment [8].

The two dynamics, represented in Fig. 2 for Tutoriallike activities and in Fig. 3 for PI, are also related to the role that each student took during discussions, which were defined in Table I based on Toulmin's argumentation pattern. 


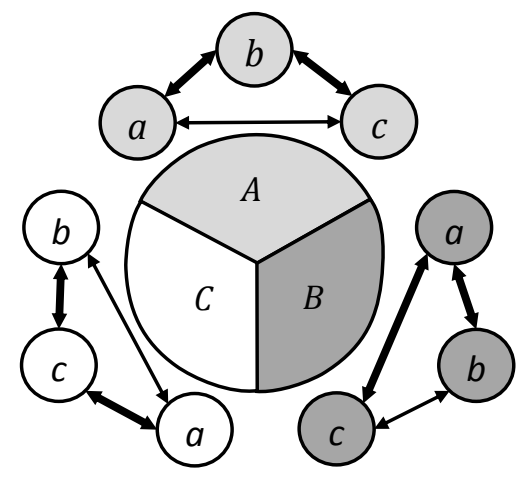

FIG. 2. Dynamics of argumentation observed during Tutorial-like activities, where students centered their discussion on each small team.

The two dynamics are influenced by these roles because, as shown in Fig. 2, in each small team there is at least one leader, $A b, B a$ and $C c$. On the other hand, during PI the leaders $A b, B a$ and $C c$ as well as the active participants $A a$, $B b$ and $B c$ engage in the discussion, while the passive participants $A c, C a$ and $C b$ refrain from participating, as shown in Fig. 3.

The differences in student's interactions during both strategies are so evident and consistent throughout the study that we can conclude that students create different dynamics of argumentation during PI compared with Tutorial-like activities, and that these dynamics are influenced by the learning environment. The dynamics are also influenced by the nature of each learning strategy, since when working with Tutorial-like activities students focus on answering a worksheet which is done in small teams, while during PI this constraint is removed and students feel free to argue within the table. Because the population is small and the study has not been repeated on other contexts, we cannot generalize the conclusion, but we can expect that this will continue to happen for the same learning environment and strategies, with different populations and courses.

Through the combination of active learning strategies like PI and Tutorial-like activities in a SCALE-UP environment, we get to observe argumentation happening at

[1] J. Osborne, Science 328, 463-466 (2010).

[2] R. Driver, P. Newton, and J. Osborne, Science Education 84, 287-312 (2000).

[3] G. Zavala and C. H. Kautz, in Proceeding of the SEFI meeting at Aalborg, Denmark, 2008.

[4] E. Brewe, L. Kramer, and V. Sawtelle, Phys. Rev. STPER 8 (1), 010101 (2012).

[5] C. H. Crouch and E. Mazur, Am. J. Phys. 69, (9) 970977 (2001).

[6] A. K. Wood, R. K. Galloway, J. Hardy, and C. M. Sinclair, Phys. Rev. STPER 10, 020107 (2014).

[7] L. C. McDermott and P. S. Shaffer, Tutorials in Introductory Physics (Prentice-Hall, Upper Saddle River, NJ, 2002).

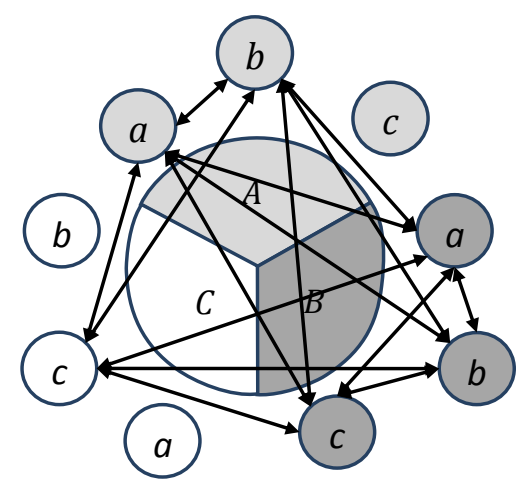

FIG. 3. Dynamics of argumentation observed during PI, where leaders and active participants centered their discussion on the table.

every level; during PI, students must think individually and then discuss within a learning community; during Tutoriallike activities, students discuss within their small teams. Finally, during both strategies students reassure their knowledge with the whole classroom.

By refraining to participate in discussions, passive students might be missing out on many opportunities to learn and expand their understanding of certain topics. It is important to identify passive students and to think of ways to encourage them to participate in discussions, so they can improve their argumentation skills. The analysis in this study can be helpful in the identification of passive students to implement strategies that help them to become more active in class by promoting their communication skills.

It would be insightful to evaluate observations of this nature with other active learning strategies in a SCALE-UP environment. These studies may give insight into the kinds of competencies that we mean to develop in our students, since different abilities are formed while working individually, collaboratively and within a learning community. It is important for students to have the opportunity to interact in several ways with different groups of people, and to confront different ways of thinking and perspectives in order to further develop their competencies of communication.

[8] R. J. Beichner and J. M. Saul, in Proceedings of the International School of Physics Enrico Fermi, Varenna, Italy, 2003.

[9] E. Mazur and R. C. Hilborn, Phys. Today 50 (4), 65. (1997).

[10] G. Zavala Enríquez, A. Domínguez Cuenca, and R. Rodríguez Gallegos, in Enfoques en Investigación e Innovación en Educación, edited by A. Domínguez Cuenca (REDIIEN, Monterrey, México, 2014), Vol. 1. p. $70-78$. 